\title{
A Simple Class of Photorheological Fluids: Surfactant Solutions with Viscosity Tunable by Light
}

\author{
Aimee M. Ketner, Rakesh Kumar, Tanner S. Davies, Patrick W. Elder, and \\ Srinivasa R. Raghavan* \\ Contribution from the Department of Chemical and Biomolecular Engineering, \\ University of Maryland, College Park, Maryland 20742-2111
}

Received July 15, 2006; Revised Manuscript Received November 5, 2006; E-mail: sraghava@eng.umd.edu

\begin{abstract}
Photorheological (PR) fluids, i.e., those with light-tunable rheological properties, may be useful in a variety of applications, such as in sensors and microfluidic devices. Currently, the need to synthesize complex photosensitive molecules hampers the applicability of these fluids. Here, we report a simple class of PR fluids that require no special synthesis and can be easily replicated in any lab from inexpensive chemicals. The fluids consist of the cationic surfactant, cetyl trimethylammonium bromide (CTAB), and the photoresponsive organic derivative, trans-ortho-methoxycinnamic acid (OMCA). Aqueous mixtures of CTAB and OMCA in basic solution self-assemble into long, wormlike micelles. Upon irradiation by UV light $(<400$ $\mathrm{nm}$ ), OMCA undergoes a photoisomerization from its trans to its cis form, which alters the molecular packing at the micellar interface. The result is to transform the long micelles into much shorter entities and, in turn, the solution viscosity decreases by more than 4 orders of magnitude. Small-angle neutron scattering (SANS) is used to confirm the dramatic reduction in micellar length. The extent of viscosity reduction in these PR fluids can be tuned based on the composition of the mixture as well as the duration of the irradiation.
\end{abstract}

\section{Introduction}

Recently, there has been much interest in creating fluids whose rheological properties (such as viscosity) are tunable by light. ${ }^{1-3}$ Such fluids could be termed photorheological (PR) fluids, ${ }^{4}$ and could offer a third paradigm for rheology modulation, following in the path of existing electrorheological (ER) fluids ${ }^{5}$ that are sensitive to electric fields and magnetorheological (MR) fluids that respond to magnetic fields. ${ }^{6} \mathrm{ER}$ and MR fluids have been around for more than 50 years, and their ability to switch rheological states has formed the basis for technologies such as dampers, clutches, and valves. ${ }^{6}$ However, these fluids are typically two-phase systems that tend to aggregate or settle with time. PR fluids, on the other hand, promise to be true, single-phase solutions, structured at the nanoscale. Also, an advantage of light over electric or magnetic fields is that light can be directed at a precise spatial location; this becomes especially valuable in microscale or nanoscale applications. Accordingly, PR fluids could be an enabling technology for microfluidic or MEMS devices and sensors.

Despite their obvious potential, PR fluids are not used widely at the moment, in either scientific labs or commercial applications. The chief reason is that current PR fluid formulations

(1) Paulusse, J. M. J.; Sijbesma, R. P. Angew. Chem., Int. Ed. 2006, 45, 23342337

(2) Eastoe, J.; Vesperinas, A. Soft Matter 2005, 1, 338-347.

(3) Tomatsu, I.; Hashidzume, A.; Harada, A. Macromolecules 2005, 38, 52235227.

(4) Wolff, T.; Emming, C. S.; Suck, T. A.; Von Bunau, G. J. Phys. Chem. 1989, 93, 4894-4898.

(5) Hao, T. Adv. Mater. 2001, 13, 1847-1857.

(6) Rankin, P. J.; Ginder, J. M.; Klingenberg, D. J. Curr. Opin. Colloid Interface Sci. 1998, 3, 373-381.

10.1021/ja065053g CCC: $\$ 37.00$ @ 2007 American Chemical Society rely on complicated photoresponsive molecules, such as, for example, a photoresponsive surfactant or polymer bearing an azobenzene moiety., ${ }^{2,-9}$ Several groups have demonstrated reversible viscosity modulation using such photosensitive moieties. The principle in each case has been to control the reversible self-assembly of the photosensitive surfactant and/or polymer between a nanostructure that yields a high viscosity (e.g., a transient network) and one that corresponds to weaker association and thereby a low viscosity. However, the difficulty in synthesizing these types of molecules has meant that the corresponding PR fluids remain accessible only to a few select research groups. There is thus a need for new PR fluids that could be readily prepared anywhere from simple, existing molecules.

In this paper, we report a new class of PR fluids that are based entirely on simple, inexpensive chemicals available to any lab. The fluids are composed of the cationic surfactant, cetyl trimethylammonium bromide (CTAB), together with a photosensitive organic acid or salt. While we have studied a number of such additives, we focus specifically on trans-ortho-methoxycinnamic acid (OMCA) in this paper. We show that CTAB/ OMCA mixtures form wormlike micelles ${ }^{10-13}$ in aqueous solution and that, upon irradiation by UV light $(<400 \mathrm{~nm})$, the

(7) Lee, C. T.; Smith, K. A.; Hatton, T. A. Macromolecules 2004, 37, 53975405.

(8) Sakai, H.; Orihara, Y.; Kodashima, H.; Matsumura, A.; Ohkubo, T.; Tsuchiya, K.; Abe, M. J. Am. Chem. Soc. 2005, 127, 13454-13455.

(9) Pouliquen, G.; Tribet, C. Macromolecules 2006, 39, 373-383.

(10) Cates, M. E.; Candau, S. J. J. Phys.: Condens. Matter 1990, 2, 68696892.

(11) Berret, J. F. In Molecular Gels; Weiss, R. G., Terech, P., Eds.; Springer: Dordrecht, 2005; pp 235-275.

(12) Raghavan, S. R.; Kaler, E. W. Langmuir 2001, 17, 300-306. 

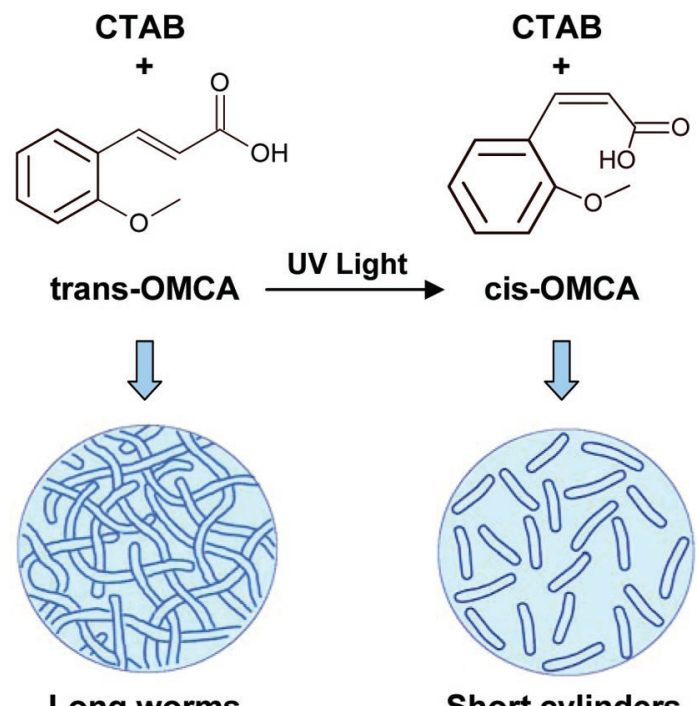

Long worms

Figure 1. Schematic behavior of photoresponsive (PR) fluids consisting of CTAB and OMCA. When OMCA is in its trans from, its mixture with CTAB gives rise to long, entangled wormlike micelles. Upon UV irradiation, trans-OMCA gets photoisomerized to cis-OMCA, and the corresponding change in molecular geometry causes a drastic reduction in micellar length.

viscosity of these solutions can be made to drop by more than 4 orders of magnitude. The basis for this viscosity change is the trans to cis photoisomerization of the double bond in OMCA (Figure 1). The resultant change in the geometry of OMCA alters the molecular packing of the CTAB/OMCA complex, leading to a drastic reduction in the length of the wormlike micelles (Figure 1). In turn, the sample is transformed from a highly viscoelastic, gel-like fluid to a thin, runny fluid with a viscosity close to that of water. Confirmation of the above microstructural hypothesis is provided by data from small-angle neutron scattering (SANS).

Micellar systems based on photosensitive additives have been investigated for a long time, following the pioneering work of Wolff and co-workers (indeed, to our knowledge, it was Wolff who coined the term "photorheological fluid").,14-16 Past studies have mainly focused on anthracene and stilbene derivatives added to cationic surfactants. However, the viscosity change induced by light in these samples was rather modest $(\sim$ a factor of $2-10){ }^{4,16}$ Thus, the principal result here is that much larger viscosity changes (factors of 1000 to 10,000) can be achieved in CTAB/OMCA mixtures. We should also note that the photosensitivity of OMCA is analogous to that of coumaric acid, which in turn is a key component of the "photoactive yellow protein". ${ }^{17}$ Although, in principle, photoisomerizations are reversible, the trans - cis isomerization of OMCA is not easily reversed, and we will discuss the reasons for this later in the paper. This means that CTAB/OMCA mixtures can only provide a one-way viscosity switch. Nevertheless, the simplicity and ease of preparation of these mixtures should make them attractive for use in some microfluidic or sensor applications. Also, the underlying principle behind this work, namely, the ability to fine-tune micellar assembly by exploiting molecular

(13) Davies, T. S.; Ketner, A. M.; Raghavan, S. R. J. Am. Chem. Soc. 2006, $128,6669-6675$.

(14) Muller, N.; Wolff, T.; von Bunau, G. J. Photochem. 1984, 24, 37-43.

(15) Wolff, T.; Klaussner, B. Adv. Colloid Interface Sci. 1995, 59, 31-94.

(16) Yu, X. L.; Wolff, T. Langmuir 2003, 19, 9672-9679.

(17) Kort, R.; Vonk, H.; Xu, X.; Hoff, W. D.; Crielaard, W.; Hellingwerf, K. J. FEBS Lett. 1996, 382, 73-78. geometry, should be applicable to similar organic additives where the photoisomerization can indeed be reversed by irradiation at a different light wavelength. We hope that this work will stimulate studies into new types of PR fluids as well as applications for these fluids in microscale devices.

\section{Experimental Section}

Materials. CTAB was purchased from Sigma-Aldrich and was greater than $98 \%$ in purity. OMCA in its trans form was purchased from Acros Chemicals, while the cis form was purchased from TCI America, and each compound was greater than $98 \%$ in purity. All chemicals were used as received. Ultrapure deionized water from a Millipore water purification system was used in preparing samples for rheological characterization, while $\mathrm{D}_{2} \mathrm{O}(99.95 \%$ deuterated, from Cambridge Isotopes) was used for the SANS studies. Solutions containing OMCA were prepared with a slight excess of base $(\mathrm{NaOH})$, and CTAB was then added to these solutions to reach the final composition. Samples were stirred continuously under mild heat until they became homogeneous. The solutions were then left to equilibrate overnight at room temperature before any experiments were conducted. The $\mathrm{pH}$ in the samples was between 9 and 11 .

Sample Response before and after UV Irradiation. CTAB/OMCA samples were irradiated with UV light from a Oriel 200 W mercury arc lamp. A dichroic beam turner with a mirror reflectance range of 280 to $400 \mathrm{~nm}$ was used to access the UV range of the emitted light. Samples $(5 \mathrm{~mL})$ were placed in a Petri dish with a quartz cover, and irradiation was done for a specific duration under stirring. Due to the nature of the OMCA spectra, irradiated samples did not undergo any changes when stored under ambient conditions, which made it easy to conduct subsequent tests using appropriate techniques such as UV-vis spectroscopy, HPLC, rheology, and SANS. UV-vis spectroscopy before and after irradiation were carried out using a Varian Cary 50 spectrophotometer.

HPLC Studies. The irradiated solutions were analyzed using HPLC (Waters Spherisorb $5 \mu \mathrm{m}$ ODS2, $4.6 \mathrm{~mm} \times 250 \mathrm{~mm}$ C18 column). A flow rate of $0.8 \mathrm{~mL} / \mathrm{min}$ was used, and the eluting solvent was $15 \%$ ethanol and $85 \%$ water. The solution components were detected using UV absorption (Dynamax absorbance detector model UV-D II) at 225 and $254 \mathrm{~nm}$. These parameters were based on those of a study of similar molecules by Imae et al. ${ }^{18}$ HPLC chromatograms of solutions before and after irradiation are provided as part of the Supporting Information.

Rheological Studies. Steady and dynamic rheological experiments were performed on an AR2000 stress controlled rheometer (TA Instruments, Newark, DE). Samples were run at $25^{\circ} \mathrm{C}$ on a cone-andplate geometry ( $40-\mathrm{mm}$ diameter, $2^{\circ}$ cone angle) or a couette geometry (rotor of radius $14 \mathrm{~mm}$ and height $42 \mathrm{~mm}$, and cup of radius $15 \mathrm{~mm}$ ). Dynamic frequency spectra were obtained in the linear viscoelastic regime of each sample as determined by dynamic stress-sweep experiments.

Solubility Studies. The solubility of OMCA isomers in water at 25 ${ }^{\circ} \mathrm{C}$ was determined as follows, much like in an earlier study..$^{13}$ An excess of the organic derivative was added to deionized water, and the solution was stirred under mild heat for 1 day, followed by equilibration at room temperature for two more days. A sample of this solution in a $1 \mathrm{~mm}$ cuvette was placed in the holder of the UV-vis spectrophotometer, maintained at $25^{\circ} \mathrm{C}$. The sample was left undisturbed for $1 \mathrm{~h}$ to allow any undissolved material to settle to the bottom of the cuvette. The absorbance was then measured and converted to a concentration value using the absorbtivity determined from a calibration curve. The same procedure was repeated for each of the OMCA isomers.

Small Angle Neutron Scattering (SANS). SANS measurements were made on the NG-7 $(30 \mathrm{~m})$ beamline at NIST in Gaithersburg, MD. Neutrons with a wavelength of $6 \AA$ were selected. Two sample-

(18) Imae, T.; Tsubota, T.; Okamura, H.; Mori, O.; Takagi, K.; Itoh, M.; Sawaki, Y. J. Phys. Chem. 1995, 99, 6046-6053. 


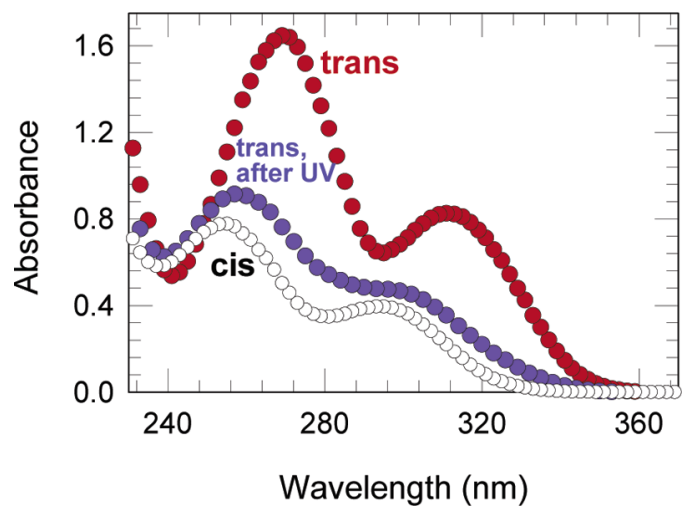

Figure 2. UV-vis spectra of trans-OMCA before irradiation, trans-OMCA after UV irradiation, and cis-OMCA. Each sample is an aqueous solution containing $1 \mathrm{mM}$ of the corresponding additive. The drop in absorbance and blue shift in the trans-OMCA curve after UV irradiation indicate that the molecule has been photoisomerized to its cis form.

detector distances of 1.33 and $13.2 \mathrm{~m}$ were used to probe a wide range of wave vectors from 0.004 to $0.4 \AA^{-1}$. Samples were studied in $2 \mathrm{~mm}$ quartz cells at $25^{\circ} \mathrm{C}$. The scattering spectra were corrected and placed on an absolute scale using calibration standards provided by NIST. The data are shown for the radially averaged intensity $I$ versus the wave vector $q=(4 \pi / \lambda) \sin (\theta / 2)$, where $\lambda$ is the wavelength of incident neutrons and $\theta$ is the scattering angle.

SANS Modeling. For dilute solutions of noninteracting scatterers, the SANS intensity $I(q)$ can be modeled purely in terms of the form factor $P(q)$ of the scatterers (i.e., the structure factor $S(q) \rightarrow 1$ in such cases). Here, we consider form factor models for two different micellar shapes: ellipsoids and rigid cylinders. ${ }^{19}$ In the expressions below, $(\Delta \rho)$ is the difference in scattering length density between the micelles and the solvent, so that $(\Delta \rho)^{2}$ is the scattering contrast. The models were implemented using software modules supplied by NIST.

A. Ellipsoids. The form factor $P(q)$ for ellipsoids of revolution with minor and major axes $R_{\mathrm{a}}$ and $R_{\mathrm{b}}$ is given by ${ }^{19}$

$$
P(q)=(\Delta \rho)^{2}\left(\frac{4}{3} \pi R_{\mathrm{a}} R_{\mathrm{b}}{ }^{2}\right)^{2} \int_{0}^{1}\left[3 \frac{(\sin z-z \cos z)}{z^{3}}\right]^{2} \mathrm{~d} \mu
$$

where $z=q \sqrt{\mu^{2} R_{\mathrm{b}}^{2}+R_{\mathrm{a}}^{2}\left(1-\mu^{2}\right)}$. Here $\mu$ is the cosine of the angle between the scattering vector $q$ and the symmetry axis of the ellipsoid.

B. Rigid Cylinders. The form factor $P(q)$ for rigid cylindrical rods of radius $R$ and length $L$ is given by ${ }^{19}$

$$
P(q)=(\Delta \rho)^{2}\left(\pi R^{2} L\right)^{2} \int_{0}^{\pi / 2}[F(q, \alpha)]^{2} \sin \alpha d \alpha
$$

where

$$
F(q, \alpha)=\frac{J_{1}(q R \sin \alpha)}{(q R \sin \alpha)} \cdot \frac{\sin (q L \cos \alpha / 2)}{(q L \cos \alpha / 2)}
$$

Here $\alpha$ is the angle between the cylinder axis and the scattering vector $q$, and $J_{1}(x)$ is the first-order Bessel function of the first kind.

\section{Results and Discussion}

We first present UV-vis spectra for trans and cis-OMCA in Figure 2. These experiments were done with aqueous solutions containing $1 \mathrm{mM}$ of the corresponding derivatives along with a slight excess of base. Figure 2 shows that the trans form has absorbance peaks at 270 and $312 \mathrm{~nm}$, whereas the peaks for the cis form occur at 254 and $293 \mathrm{~nm}$. Note that the trans form has much higher absorbances than the cis form. Thus, irradiation

(19) Pedersen, J. S. Adv. Colloid Interface Sci. 1997, 70, 171-210.
(A) Before UV Irradiation

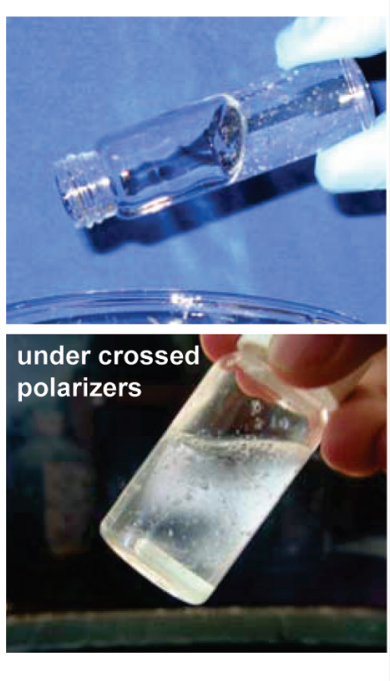

(B) After UV Irradiation

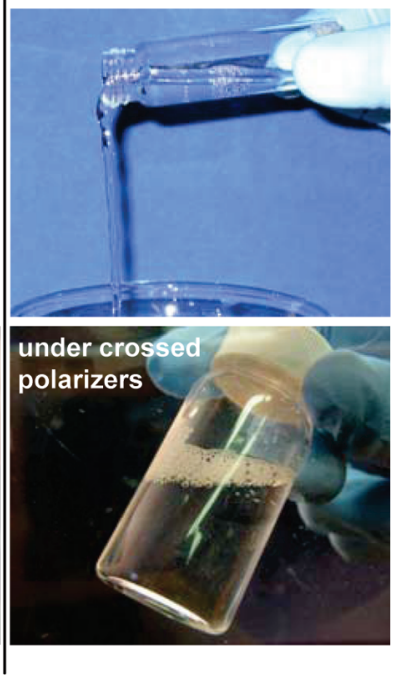

Figure 3. Photographs of a $60 \mathrm{mM} \mathrm{CTAB}+50 \mathrm{mM}$ OMCA sample (A) before and (B) after UV irradiation. (A) Initially, OMCA is in its trans form, and the sample is highly viscoelastic due to the presence of long worms. Consequently, the sample does not flow even when the vial is tilted (top) and retains bubbles for a long time (bottom). Moreover, the sample shows flow-birefringence; i.e., when viewed under crossed polarizers, streaks of light appear when the sample vial is lightly shaken (bottom). (B) Upon UV irradiation, OMCA is isomerized to its cis form, leading to much shorter micelles. In turn, the sample viscosity is significantly reduced, as shown by its rapid pourability (top) and by the rapid rise of bubbles to the liquid surface (bottom). Also, the flow-birefringence is no longer observed (bottom).

of the trans derivative with UV light will cause the molecule to absorb light, and this, in turn, can trigger a photoisomerization to its cis state, very similar to what occurs for other cinnamic acid derivatives. To test this, we irradiated the trans-OMCA solution with UV light and recorded UV-vis spectra after irradiation. As shown in Figure 2, UV irradiation causes a blue shift in the UV spectrum of trans-OMCA, with the peaks shifting to 257 and $300 \mathrm{~nm}$ (the peak heights are also significantly reduced). This blue shift is indicative of a trans to cis photoisomerization. Importantly, the shift in the spectrum can be effected by just 1 min of UV irradiation, and no further changes in the spectrum occur with longer irradiation times. The irradiated sample thus corresponds to a photostationary equilibrium of mostly cis (ca. 83\%) molecules.

We now consider the effects of UV irradiation on mixtures of OMCA with the cationic surfactant, CTAB. Henceforth, the abbreviation OMCA will be used to refer to its trans form, which is the common, commercially available isomer. (On the other hand, cis-OMCA will be specifically denoted as such.) Incidentally, the presence of CTAB has no effect on the UV spectra of OMCA and thereby on the UV-induced trans to cis photoisomerization. Our initial studies with CTAB/OMCA mixtures showed that these could form highly viscous and viscoelastic solutions. For example, Figure 3 shows photographs of a sample containing $60 \mathrm{mM} \mathrm{CTAB}$ and $50 \mathrm{mM}$ OMCA. This sample is viscous enough that it does not flow readily out of a tilted vial (Figure 3A). Also, when bubbles are introduced by shaking the vial, they remain trapped in the fluid for long periods of time. The sample also shows flow-birefringence; i.e., when viewed under crossed polarizers, streaks of light appear upon gently shaking or tapping the vial (Figure $3 \mathrm{~A}) .{ }^{11}$ Taken together, 
(a) Before UV Irradiation

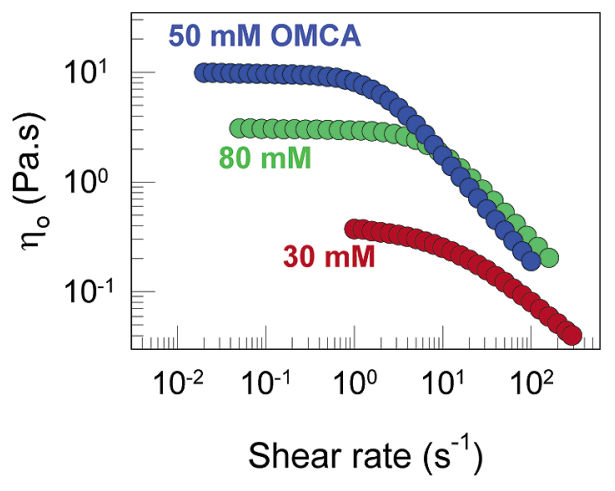

(b) After UV Irradiation

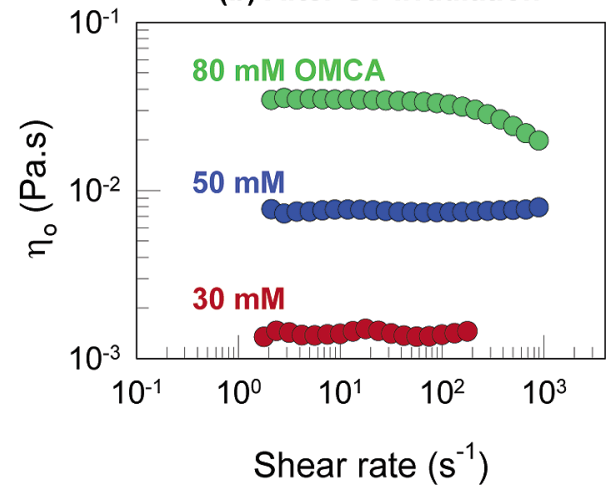

Figure 4. Viscosity vs shear rate plots for three CTAB/OMCA mixtures (a) before and (b) after UV irradiation for 30 min. The samples each contain 60 mM CTAB, and their OMCA concentrations are indicated on the plots. All samples show a shear-thinning response before irradiation, whereas, after irradiation, the samples are mostly Newtonian with much lower viscosities.

(a) Before UV Irradiation

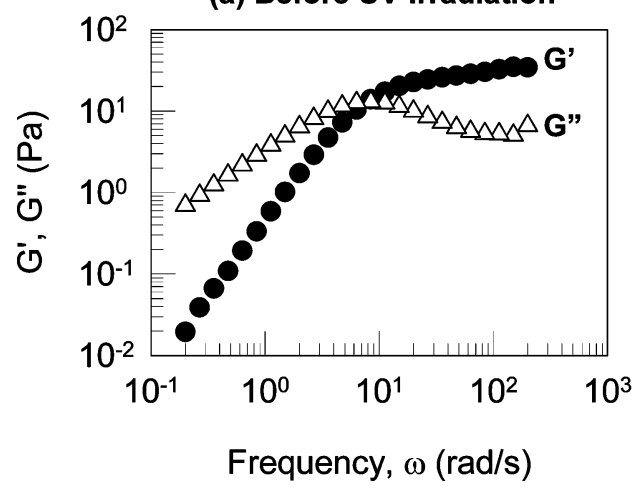

(b) After UV Irradiation

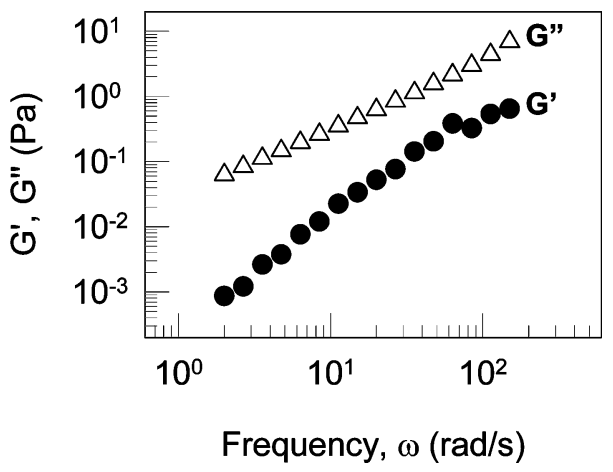

Figure 5. Dynamic rheology of a $60 \mathrm{mM} \mathrm{CTAB}+80 \mathrm{mM}$ OMCA sample (a) before and (b) after UV irradiation for 30 min. Before irradiation, the sample shows a viscoelastic response, whereas, after irradiation, its response is predominantly viscous.

the viscoelasticity and flow-birefringence suggest the presence of wormlike micelles, i.e., long, flexible, cylindrical chains in the CTAB/OMCA sample. ${ }^{11,13}$ On the other hand, CTAB/cisOMCA mixtures are visually quite different. Those samples are low-viscosity, watery solutions that do not show any flowbirefringence (viscosity data for these mixtures will be presented later in Figure 7).

Based on the different rheological responses induced by the trans and cis forms of OMCA in CTAB mixtures, we were interested in examining the effects of light irradiation on sample rheology. Accordingly, we irradiated the $60 \mathrm{mM} \mathrm{CTAB}+50$ mM OMCA with UV light for $30 \mathrm{~min}$. Figure 3B presents visual evidence for the effects induced by UV irradiation on the sample rheology. The photographs show that the sample has been converted to a thin, runny fluid that can be easily poured out of the vial and in which bubbles rapidly rise to the surface. Moreover, the irradiated sample does not show flow-birefringence. Thus, Figure 3 demonstrates a dramatic light-induced rheological transition in the CTAB/OMCA sample. Based on the spectra in Figure 2, the rheological change is clearly a result of the trans to cis photoisomerization of OMCA. We will show that this change in molecular geometry causes a dramatic reduction in micellar length, which explains the drop in viscosity.

We now present rheological data on selected CTAB/OMCA samples to quantify the light-induced rheological changes. Note that, following irradiation, CTAB/OMCA samples remain unaltered when stored under ambient conditions (exposure to visible light has no effect because OMCA has a negligible absorbance in the visible range of the spectrum). Thus, irradiated samples could be tested subsequently on the rheometer. Figure 4 shows steady-shear rheological data (viscosity vs shear rate) for three samples, each containing $60 \mathrm{mM}$ CTAB, with OMCA concentrations of 30,50 , and $80 \mathrm{mM}$, respectively. Before irradiation (Figure 4a), all three samples show shear-thinning behavior, with a plateau in the viscosity at low shear rates, followed by a decrease in viscosity at higher shear rates. The zero-shear viscosity $\eta_{0}$ is highest for the $50 \mathrm{mM}$ OMCA sample, with a value of about $10 \mathrm{~Pa} \cdot \mathrm{s}$ (i.e., about 10,000 times the viscosity of water). With further increases in OMCA content, there is a drop in $\eta_{0}$ (this is further discussed under Figure 7), and the $80 \mathrm{mM}$ OMCA sample has an $\eta_{0}$ value of about $2 \mathrm{~Pa}$. s. Figure $4 \mathrm{~b}$ shows the rheology of the same three samples after UV irradiation for $30 \mathrm{~min}$. The irradiated samples show negligible shear rate-dependence of their viscosities; i.e., their behavior is mostly Newtonian (only the $80 \mathrm{mM}$ OMCA sample shows slight shear-thinning). Moreover, the viscosities are much lower compared to Figure 4a, with each sample showing a drop in $\eta_{0}$ by several orders of magnitude due to UV irradiation. The measurements thus confirm the visual observations reported in Figure 3.

In addition to steady-shear rheology, it is useful to examine the rheological response under dynamic or oscillatory shear, which is a more sensitive probe of the nanostructure in complex fluids. Figure 5 shows dynamic rheological data on a sample containing $60 \mathrm{mM} \mathrm{CTAB}$ and $80 \mathrm{mM}$ OMCA. The data are 


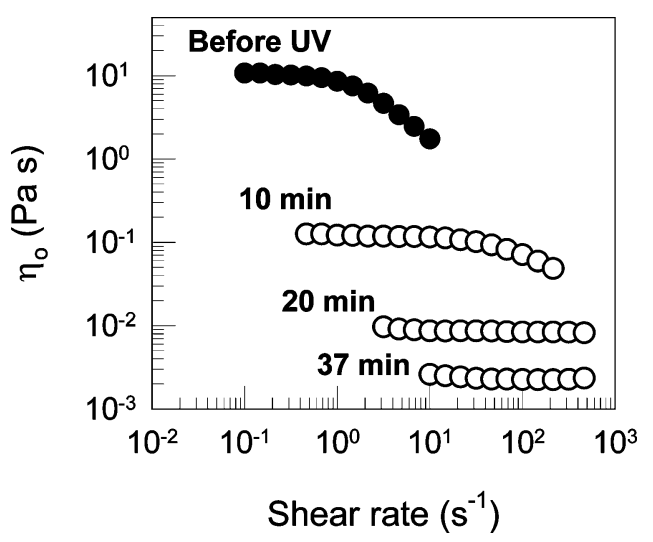

Figure 6. Steady-shear rheology of a $60 \mathrm{mM} \mathrm{CTAB}+50 \mathrm{mM}$ OMCA sample before irradiation and after UV irradiation for various periods of time (as indicated on the plot). The sample is observed to switch from a highly viscous, shear-thinning fluid to a low-viscosity, Newtonian fluid with progressive irradiation.

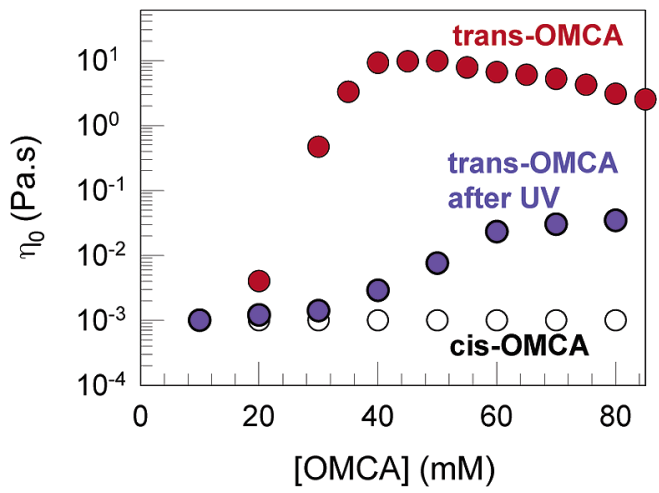

Figure 7. Zero-shear viscosity $\eta_{0}$ of $60 \mathrm{mM} \mathrm{CTAB}+$ OMCA mixtures as a function of the OMCA content. Data are shown for samples containing trans-OMCA, for cis-OMCA, and for trans-OMCA samples after $30 \mathrm{~min}$ of UV irradiation. A significant light-induced drop in viscosity is observed for the trans-OMCA samples.

presented as plots of the elastic modulus $G^{\prime}$ and viscous modulus $G^{\prime \prime}$ as functions of the angular frequency $\omega$. Before irradiation (Figure 5a), the sample shows a typical viscoelastic response; i.e., the behavior is elastic $\left(G^{\prime}>G^{\prime \prime}\right.$, plateau in $\left.G^{\prime}\right)$ at high frequencies or short time scales, while it is viscous $\left(G^{\prime \prime}>G^{\prime}\right.$, both moduli are strong functions of frequency) at low frequencies or long time scales. On the other hand, after irradiation with UV light for $30 \mathrm{~min}$ (Figure 5b), the sample exhibits a purely viscous response over the entire range of frequencies. Thus, dynamic rheology confirms a light-induced transition from a viscoelastic fluid to a thin, viscous one, again consistent with the photographs in Figure 3.

We have thus shown that appreciable rheological changes can be induced in CTAB/OMCA samples by UV irradiation. Next, we discuss the evolution of sample rheology as a function of irradiation time. Figure 6 shows viscosity vs shear rate plots for a $60 \mathrm{mM} \mathrm{CTAB}+50 \mathrm{mM}$ OMCA sample after various periods of UV irradiation. Before irradiation, the sample is highly viscous and strongly shear-thinning. After $10 \mathrm{~min}$ of irradiation, the zero-shear viscosity is reduced by about 2 orders of magnitude and the extent of shear-thinning is also reduced. After $20 \mathrm{~min}$, the viscosity is further reduced and the sample behaves like a Newtonian fluid over the shear rates investigated. Finally, after $37 \mathrm{~min}$, the viscosity drops to that of the solvent, i.e., water. No further changes in viscosity occur with longer irradiation. The data show that the viscosity drop can be controlled through the irradiation time. It is worth pointing out here that the rate of viscosity reduction is determined primarily by the rate of absorption of UV light by the sample, which in turn depends on the intensity of the UV lamp, the sample volume used, and the experimental geometry (path length). Once light is absorbed by the OMCA molecules, they will photoisomerize on a time scale of milliseconds. In the above experiments, we did not attempt to optimize the transition speed, but it is easy to achieve faster viscosity transitions with CTAB/OMCA fluids. Indeed, preliminary experiments with smaller sample volumes show that a significant viscosity drop can be achieved within a few seconds.

The viscosity drop upon UV irradiation can also be controlled via the sample composition itself. Figure 7 shows the zeroshear viscosity $\eta_{0}$ of $60 \mathrm{mM}$ CTAB solutions as a function of either trans-OMCA or cis-OMCA concentration. For the transOMCA samples, data are also shown after $30 \mathrm{~min}$ of UV irradiation. Note that the viscosities of CTAB/cis-OMCA solutions are very low and approximately identical to that of water. On the other hand, the viscosities induced by transOMCA are much higher and reach a peak around $50 \mathrm{mM}$ of the additive (such a viscosity peak vs additive concentration is often seen for wormlike micelles ${ }^{11,12}$ and is believed to signify a transition from linear to branched worms). Upon UV irradiation of the trans-OMCA samples, the viscosities drop in all cases, with the extent of the drop being highest (ca. 10,000fold) near the peak. It should be noted that, for all samples, irradiation for longer times will cause the viscosity to drop further until the photoisomerization is complete. At this stage, the viscosity will drop to that of the cis-OMCA samples, i.e., to a waterlike viscosity.

The above rheological and visual observations on CTAB/ OMCA samples indicate a transition from long, wormlike micelles to much shorter micelles upon UV irradiation. To recap, before irradiation, the samples are flow-birefringent, they are viscoelastic under dynamic rheology, and shear-thinning in steady rheology. All these phenomena are associated with wormlike micelles: the flow birefringence arises because the worms tend to align along the flow direction when sheared, the viscoelastic behavior is due to the entanglement of the worms into a transient network, and the shear-thinning is caused by a disruption of this network due to shear. ${ }^{11,13}$ The absence of these phenomena in the irradiated sample suggests that the micelles have been reduced to much shorter (nonentangled) structures. In order to independently verify such a reduction in micellar size, we turn to a second technique, namely SANS.

Samples for SANS were made in $\mathrm{D}_{2} \mathrm{O}$ to achieve the required contrast between the micellar structures and solvent. We verified that CTAB/OMCA samples in $\mathrm{D}_{2} \mathrm{O}$ were rheologically identical to those made in $\mathrm{H}_{2} \mathrm{O}$. SANS data can be readily interpreted in terms of micelle size and shape only when intermicellar interactions (structure factor contributions) are minimized, i.e., when electrostatic interactions are screened and the micelle volume fraction is low. We therefore studied equimolar CTAB/ OMCA samples at relatively low concentrations. SANS spectra ( $I$ vs $q$ ) are shown in Figure 8 for three samples with CTAB/ OMCA concentrations of 30/30, 20/20, and 5/5 mM, respectively. In each case, data are shown before and after UV irradiation for $30 \mathrm{~min}$. We note that, for all samples, irradiation 
(a)

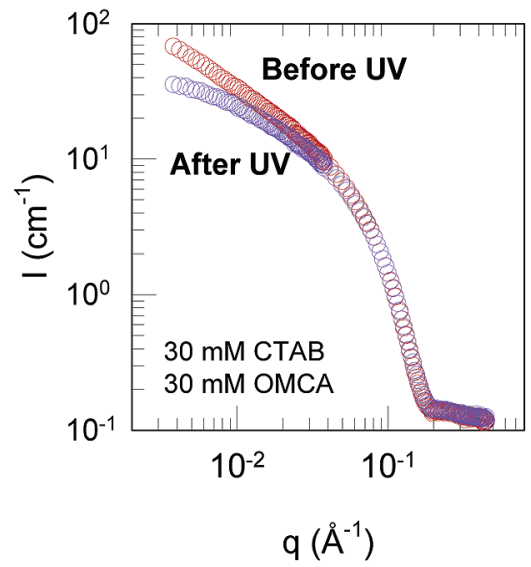

(b)

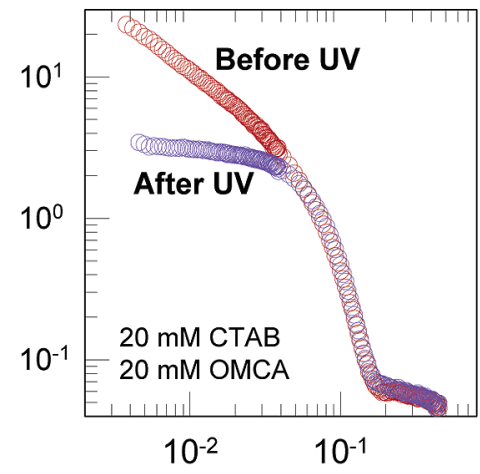

$q\left(\AA^{-1}\right)$ (c)

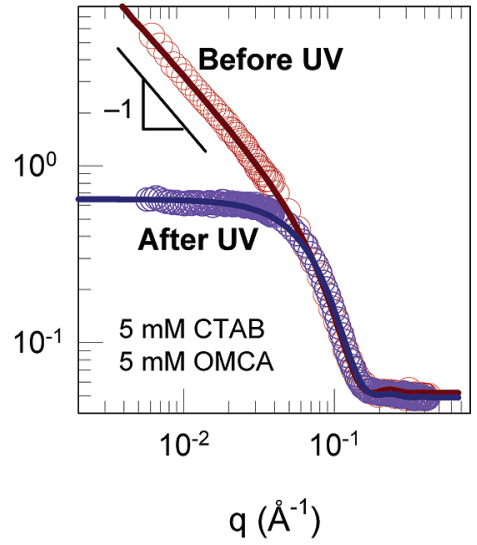

Figure 8. SANS scattering spectra from three CTAB/OMCA mixtures before and after 30 min of UV irradiation. The lines through the data for the $5 / 5$ CTAB/OMCA sample are model fits to cylindrical micelles (before irradiation) and ellipsoidal micelles (after irradiation).

causes a significant drop in the scattered intensity at low $q$. The drop in intensity is a direct, qualitative indication of a decrease in micelle size, which is consistent with our expectation.

To obtain a more quantitative measure of micellar sizes, we model the SANS data from the $5 / 5$ sample. For this sample (Figure 8c), a large decrease in intensity is seen upon UV irradiation, presumably because the micelle sizes fall within the window probed by SANS. Prior to irradiation, the intensity asymptotes at low $q$ to a slope of about -1 , which is indicative of long, cylindrical structures. ${ }^{19}$ We therefore model the micelles as rigid cylinders (eqs 2, 3), and the fit is shown as a solid line through the data. From the fit, the micellar radius is obtained to be about $22 \AA$, while their length is ca. $3000 \AA$. The same sample after irradiation shows a plateau in SANS intensity at low $q$, which suggests the presence of smaller and more globular structures. Accordingly, we model the micelles in this case as ellipsoids of revolution (eq 1), and the corresponding fit is again shown as a solid curve through the data. A good fit is obtained for prolate ellipsoids with radii of 22 and $40 \AA$, respectively, for their major and minor axes. Thus, as expected, there is a dramatic reduction in the largest dimension of the micelles (a factor of about 100) due to UV irradiation.

The SANS data thus confirm that the light-induced viscosity reduction in $\mathrm{CTAB} / \mathrm{OMCA}$ mixtures is due to a decrease in micelle size. The micellar size reduction, in turn, is evidently caused by the photoisomerization of OMCA from trans to cis, as indicated by the UV-vis spectra (Figure 2). Note that there is negligible evidence for other light-induced effects such as photodimerization in our samples (the latter has been shown to occur in certain mixtures of cinnamic acid and zwitterionic surfactants ${ }^{18}$ ). Indeed, HPLC studies on our solutions (see Supporting Information) confirm that photoisomerization of OMCA is the dominant light-induced effect. This hypothesis is consistent with the results of Figure 7, which show that transand cis-OMCA differ greatly in their ability to induce growth of CTAB micelles.

The question then is why are trans- and cis-OMCA so different? Can we rationalize these differences based on what we know about wormlike micelles and their self-assembly? We believe that there are two related factors to consider in this regard: (i) the geometry of the two OMCA isomers and (ii) their hydrophobicities. Both these factors will influence the association of OMCA with CTAB micelles. In turn, the association of OMCA will reduce the micellar surface charge, thus decreasing the effective area of the charged headgroups and, thereby, facilitating the growth of long, cylindrical micelles. In other words, the key requirement for micellar growth is the association (adsorption) of OMCA counterions at the micellar interface. ${ }^{11}$ Much like other aromatic counterions, OMCA is expected to adsorb in such a way that the hydrophobic aromatic ring is embedded in the hydrophobic interior of the micelle while the carboxylate anion is located next to the cationic headgroups at the interface. ${ }^{13,20}$ Also, the extra methyl group on OMCA must be preferably oriented toward the micelle interior as well. Such an orientation appears to be achievable for trans-OMCA but more difficult for cis-OMCA (Figure 1). In the case of cisOMCA, the methyl and carboxylate moieties are brought into close proximity such that it will be difficult for the former to orient inward and the latter outward relative to the micellar interface. In short, geometric arguments suggest that transOMCA will strongly associate with CTAB micelles, whereas cis-OMCA will associate weakly.

A second related argument concerns the relative hydrophobicities of the two isomers: specifically, we believe that transOMCA is more hydrophobic than cis-OMCA. A greater hydrophobicity of the trans isomer has been noted for a variety of compounds, including olefinic and azobenzene derivatives. For example, in azobenzene-based surfactants, the greater hydrophobicity of the trans form gives it a lower critical micelle concentration or CMC. ${ }^{7,8}$ This difference in hydrophobicity has been attributed to the lower net dipole moment of the trans compared to the cis isomer: i.e., the dipoles add up in the cis, while they tend to cancel each other in the trans (equivalently, there is greater charge delocalization in the $\mathrm{cis}$ form) ${ }^{7,8}$ Moreover, our own studies further confirm the differences in hydrophobicity. In the absence of base, we find that trans- and cis-OMCA have limited solubility in deionized water. But while the solubility of cis-OMCA is measured to be $8.6 \mathrm{mM}$, the solubility of trans-OMCA is just $0.26 \mathrm{mM}$, i.e., more than a factor of 30 less. Accordingly, our solubility measurements confirm that trans-OMCA is much more hydrophobic than cisOMCA, which suggests that the former will also remain associated with micelles to a much greater extent. Indeed, a lower solubility in water has been correlated with a greater

(20) Hassan, P. A.; Raghavan, S. R.; Kaler, E. W. Langmuir 2002, 18, $2543-$ 2548 . 
association with micelles previously. ${ }^{13}$ All in all, the hydrophobicity arguments support the geometric reasoning advanced above.

Our arguments ultimately lead to the following mechanism to explain our results: when trans-OMCA is mixed with $\mathrm{CTAB}$, the counterions will strongly associate with CTAB micelles, causing micellar growth. Upon UV irradiation, trans-OMCA is converted to cis-OMCA, which has a much weaker interaction with $\mathrm{CTAB}$, and will therefore tend to desorb from the micellar interface. As a result, the effective area of the CTAB headgroups increases, inducing the micelles to transform into much smaller structures and, thereby, driving the sharp drop in solution viscosity.

Last, it is worth discussing the generality of our results as well as the reversibility of the viscosity changes. We have observed similar light-induced viscosity transitions with several aromatic derivatives similar to OMCA, including cinnamic acid and ortho-coumaric acid. Thus, the results and mechanism presented here should extend to a range of aromatic derivatives. With regard to reversing the viscosity changes, we note from the UV-vis spectra that trans-OMCA has a much higher absorption than cis-OMCA over the entire wavelength range, which precludes a reverse cis to trans photoisomerization (but an acid-catalyzed reversal is possible ${ }^{21}$ ). Nevertheless, a variety of other organic molecules do permit reversible photoisomerizations, ${ }^{15}$ and the key will be to employ a molecule whose photoisomers have different effects on micellar self-assembly. Further work in this context is ongoing in our labs.

\section{Conclusions}

We have demonstrated light-responsive rheological properties in a class of micellar fluids consisting of the cationic surfactant, $\mathrm{CTAB}$, and the organic derivative, OMCA. When OMCA is in its trans form, it associates strongly with CTAB, leading to the formation of long, wormlike micelles. These micelles entangle into a transient network and thereby give rise to a highly viscoelastic fluid. Upon irradiation by UV light, trans-OMCA is photoisomerized to cis-OMCA. The cis isomer has a much weaker interaction with $\mathrm{CTAB}$ since its geometry does not favor binding at the micellar interface and also because it is more hydrophilic. Consequently, the $\mathrm{cis}$ isomer tends to desorb from the micellar interface, transforming the micelles into much smaller structures. As a result, the sample is converted into a low-viscosity, Newtonian fluid. A drop in zero-shear viscosity by several orders of magnitude can be induced in these fluids upon light irradiation for a few minutes. These photorheological (PR) fluids could thus be promising for use in microscale flowcontrol devices and sensors.

Acknowledgment. This work was funded by a seed grant from the Small Smart Systems Center (SSSC) at UMD and a CAREER award from NSF-CTS. Undergraduate students, Jamie Chandler and Joanna Tinnirella, helped with some of the rheology experiments. We are grateful to Angela Lewandowski and Professor Bill Bentley for help with the HPLC measurements. Finally, we would like to acknowledge NIST for facilitating the SANS experiments performed as part of this work.

Supporting Information Available: Details on sample characterization using HPLC are described in this section of the paper. This information is available free of charge via the Internet at http://pubs.acs.org.

JA065053G

(21) Noyce, D. S.; King, P. A.; Kirby, F. R.; Reed, W. L. J. Am. Chem. Soc. 1962, $84,1632-1635$. 


\title{
Supporting Information for
}

\author{
A Simple Class of Photorheological Fluids: Surfactant Solutions with \\ Viscosity Tunable by Light
}

\author{
Aimee M. Ketner, Rakesh Kumar, Tanner S. Davies, Patrick W. Elder, and \\ Srinivasa R. Raghavan* \\ Department of Chemical \& Biomolecular Engineering \\ University of Maryland \\ College Park, MD 20742-2111 \\ *Corresponding author. email: sraghava@eng.umd.edu
}

\section{$\underline{\text { Results and Discussion: HPLC }}$}

We used HPLC to determine whether the effect of light on OMCA involved a combination of photoisomerization (trans to cis) as well as photodimerization. This technique has been used earlier by Imae et al. ${ }^{1,2}$ to identify the presence of photodimers in the case of cinnamic acid, as well as the relative amounts of photodimers compared to photoisomers. HPLC was performed on samples of CTAB/trans-OMCA and CTAB/cisOMCA prior to UV irradiation, as well as CTAB/trans-OMCA following UV irradiation. A mixture of ethanol and water at a ratio of 15:85 was used as the mobile phase of the HPLC and the flow rate was maintained at $0.8 \mathrm{ml} / \mathrm{min}$. Typical HPLC chromatograms for the three samples are shown in the figure below. Note that the CTAB/trans-OMCA sample after UV-irradiation (bottom plot) only shows three peaks, which correspond to $\mathrm{NaOH}$, cis-OMCA, and trans-OMCA respectively, in order of increasing elution times. This sample was run in the HPLC column for 2 hours and no other peaks emerged at elution times beyond 15 minutes. Based on the earlier work of Imae et al., 1,2 if dimers had been formed by irradiation, they would have eluted from the column within a few minutes after the trans-OMCA. Note also that the height of the cis peak in the chromatogram is much greater than the trans peak, thereby demonstrating that a trans to cis photoisomerization has indeed occurred in this sample.

\section{References:}

1. Imae, T.; Tsubota, T.; Okamura, H.; Mori, O.; Takagi, K.; Itoh, M.; Sawaki, Y. J. Phys. Chem. 1995, 99, 6046-6053.

2. Takagi, K. ; Nakamura, T.; Katsu, H.; Itoh, M.; Sawaki, Y.; Imae, T. Mol. Cryst. Liq. Cryst. 1996, 276, A135-A138. 

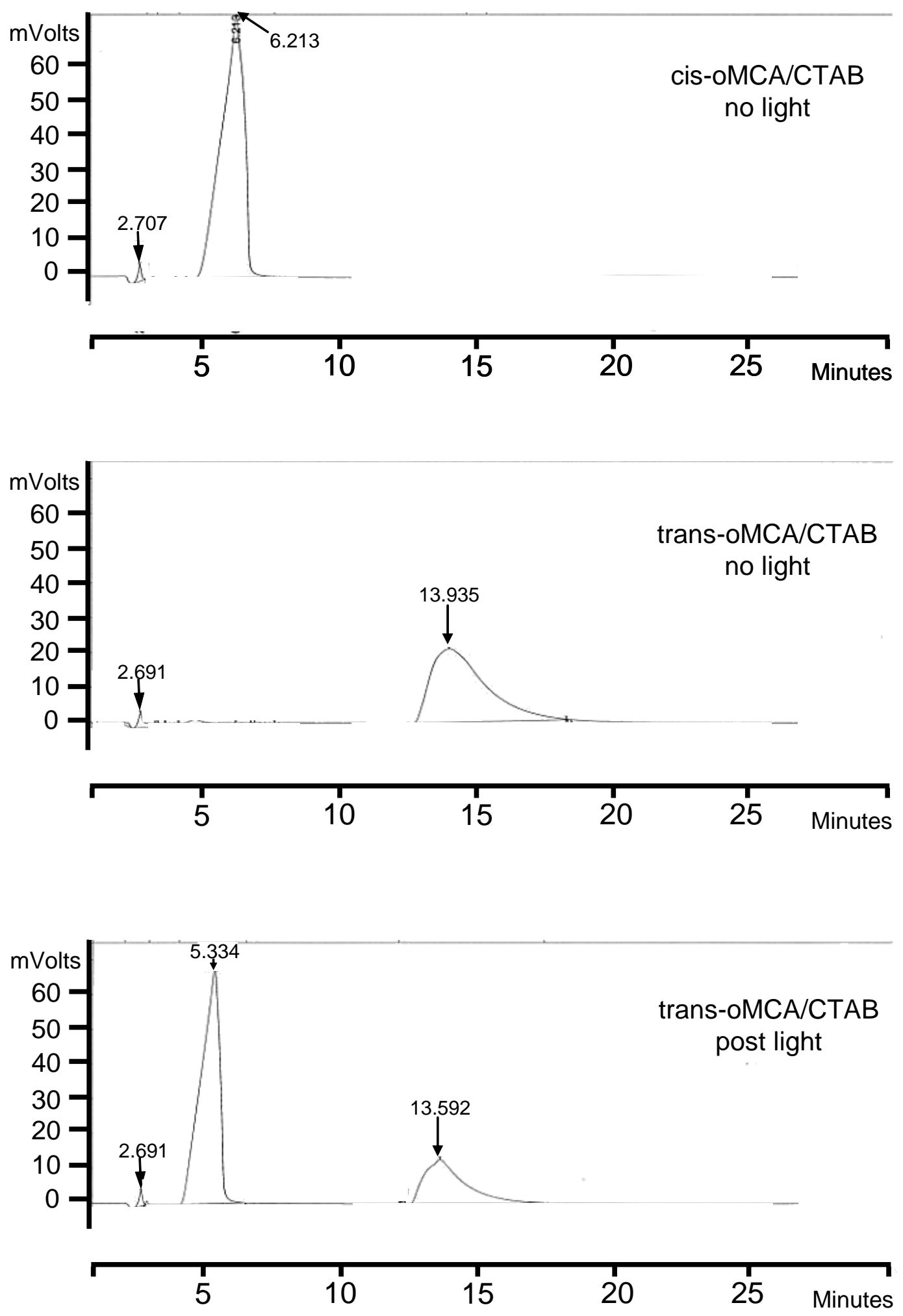